\title{
Effects of Covid and its Preventive Measures
}

\author{
Ankita Chopra \\ Assistant Professor Jagan Institute \\ of Management \\ Studies \\ Rohini, Sector 5, Delhi
}

\author{
Ritika \\ Student \\ Jagan Institute of Management \\ Studies \\ Rohini, Sector 5, Delhi
}

\author{
Vani Malhotra \\ Student \\ Jagan Institute of Management \\ Studies \\ Rohini, Sector 5, Delhi
}

\begin{abstract}
This paper investigates the impacts of corona virus pandemic (COVID-19) and the vaccination required, as well as prospective policy directions to limit its effects, based on a comprehensive survey of the literature. According to our findings, the Covid-19 pandemic has had negative consequences on humanity, the human body, and people of various age groups, in addition to mortality. This 2019 novel corona COVID19 is a highly contagious zoonosis caused by SARSCoV2 and spread from person to person by respiratory secretions. The elderly and persons with comorbidities are the most vulnerable populations who require ventilation. People over the age of 55 to 65 who have a history of chronic diseases (uncontrolled hypertension, diabetes, COPD, and all other clinical disorders) should avoid contact with potentially infected patients. Furthermore, the survey indicates the safe and effective vaccination and its preventive measures. Finally, this research looks into future research directions.
\end{abstract}

\section{Keywords}

SARS-COV-2, COVID-19, Vaccination, Covaxin/Covishield, Comorbidity, Preventive Measures

\section{INTRODUCTION}

SARS CoV2 appears to have created the transfer from wildlife to mankind in the Wuhan's Huanan seafood market, China. [1]. According to Aaron Irving, an infectious-diseases researcher at Zhejiang University in Haining, China, the findings suggest that more SARS- CoV-2 cousins may be held in lab freezers. Irving also aims to examine stored samples of bats and other mammals for antibodies against SARSCoV2. Further corona-viruses discovered in other pangolins and Rhinolophus bats captured between 2015 and 2019 are now known to be closely related to SARS-CoV-2, according to scientists. The findings, according to Latinne, prove that Rhinolophus bats are the virus's reservoir. To be a direct ancestor of the current pandemic virus, the new virus would have to be 99 percent related to SARS-CoV-2, according to Irving. More Coronaviruses linked to SARS- CoV-2 are most likely present in Rhinolophus bat populations. [2].

\section{LITERATURE REVIEW}

The purpose of this conceptual paper is to discuss the findings of a study on COVID-19 and the precautions (vaccination) that must be taken to keep safe. This survey provides an overview of (i) the Covid affecting Humanity ;

(ii) the Covid affecting Human Body; (iii) the Covid affecting people of different Age Groups; and (iv) the preventive measures i.e safe and effective allocation of vaccines to more vulnerable age groups of variant-1.

Pathogens like the Zika virus, Ebola virus, coronaviruses and Nipah virus have developed in a range of diverse places in recent decades, with infections like the Zika virus, Ebola virus, coronaviruses and Nipah virus among them(CoVs). In Wuhan, China, a new kind of infection with a virus has been detected, and preliminary genome sequencing data suggests that the virus is a new strain of $\operatorname{CoV}(2019-\mathrm{nCoV})$, named severe acute radiation illness CoV-2(SARSCoV2). Despite the fact that corona-virus disease 2019 (COVID19) is likely to have started in an host animal (zoonotic origination) before spreading from from one person to another, other possibilities should not be discounted. COVID-19 has a milder pathophysiology than other human CoVs, but it has a higher transmission capacity, as indicated by the growing number of cases that have been confirmed all across the globe. In comparison to additional newly discovered viruses such as avian H7N9, SARS-CoV, Ebola and Middle East respiratory syndrome coronavirus, SARS-Cov-2 has a low virulence and moderate transmissibility (MERS-CoV). This unique virus was transmitted derived from an animal, like bats, according to codon usage analysis. Early detection using RTPCR and further generation synchronization has aided in the early detection of the infection[3].The corona-virus 2019(CoVid19) was very first detected in individuals with serious pulmonary sickness in Wuhan of China. The cause was a new coronavirus called as severe acute respiratory syndrome coronavirus 2 (SARS-CoV-2). A lot more 2,850,000 people have been contaminated since its discovery, with approximately 200,000 of them dying [1,4,5]. COVID-19 has worrying traits, such as its apparent ease of spread and penchant for producing severe disease in the elderly and those with pre-existing medical issues [6,7]. Furthermore, coronaviruses are known for their ability to change and recombine. SARS-genomic research Since its discovery, CoV-2's sequence has changed. According to some specialists, these changes have boosted the virus's virulence, and two strains are currently in circulation: the fatal " $\mathrm{L}$ " and the less virulent "S"[8,9]. At this moment, there have been no effective antibiotic medicines or immunizations present for CoVid-19. Therapies are mostly focused on assistance for symptoms and respiratory issues, as dictated by the health authorities in each country's protocols, with many nations adhering to the WHO protocol[6,7]. Almost all patients with severe symptoms are willing to use oxygen. In life-threatening conditions, as a rescue treatment, passive vaccination with recovered plasma and immunoglobulin $\mathrm{G}$ transfusion can be employed. [10,37]. In view of the current scenario, finding a cure has become a top goal for the world's health authorities. Several firms are developing a therapy or vaccination for SARS-CoV2. This is not at all, however, a rapid procedure; experts expect it will take between 12 and 18 -month period.

Covid-19 is the third new coronavirus in the twenty-first century to produce a large-scale pandemic, followed by the SARS Corona-virus (SARS-CoV) outbreak in 2003 and the Middle East Respiratory Syndrome Coronavirus (MERS-CoV) outbreak in $2012[11,12]$. We can use our knowledge and experience from the last two pandemics to develop therapeutic solutions for this one. Various therapies and pharmacological combinations have lately shown to be effective. Take, the combination of chloroquine with azithromycin, for example. Additionally, malaria and the antiviral Remdesivir medication chloroquine has gotten a lot of attention as potential COVID-19 treatment all around the world.. 
On the other hand, other businesses are focusing on developing a treatment using a novel technique, such as antiviral RNA. $[12,13]$.

\section{COVID AFFECTING HUMANITY}

The world is engulfed in a worldwide health emergency that is wreaking havoc on humanity's health. SARS-CoV2 is a worldwide public health danger. A sociological danger is a natural or man-made hazard which has the potential to negatively impact a large amount of the population of humans. As of November 4 2020, the Covid-19 pandemic had infected more above 46 million people in 235 countries and areas around the globe, stated by the World Health Organization(2020). It has left a trail of immense devastation in its wake, and its impact extends beyond the health of humanity's current generation. According to the World Bank, the number of people living in extreme (those living on a small budget of $\$ 1.90$ per day) would rise by 150 million by 2021. Poverty suffocates social development and future successors' potential to thrive. The whole human cost of COVID-19 is enormous. [14].

\section{COVID AFFECTING HUMAN BODY}

Covid 19 has an effect on the human respiratory tract. The illness begins with flu-like symptoms or no symptoms at all, then escalates to more serious symptoms. Covid 19 mostly targets the lungs of patients who are sick, causing ARDS and pneumonia in extreme situations. It's important to remember that ARDS and pneumonia aren't invariably the result, though they are a possibility in the most serious cases. In the great majority of cases, 80 percent will have minimal side effects, 14 percent will develop pneumonia, 5 percent will have sepsis and organ failure (majorly respiratory failure), and $2 \%$ will be deadly. Few of the major signs to look at in a Covid-19 affected person include sickness, disorientation, dyspnea, headache, dry cough (which leads to phlegm), and, loss of smell and taste in some cases. In few cases, diarrhoea and exhaustion was reported. While a Covid-19 infected person can be treated, it's crucial to remember that underlying conditions (hypertension, diabetes, cardiac problems, pulmonary disorders) and persons taking immunesuppressing medicines are among the reasons that can lead to death. The danger is increased in older people since their immunity declines with age and they are more susceptible to various ailments. Understanding Covid 19's impact on the bodily system, particularly the lungs, is very critical. Covid 19 has a direct effect on the lungs, causing destruction to the alveoli (tiny air sacs). Role of the alveolus is carrying oxygen to the blood vessels. The blood arteries, or capillaries, supply oxygen to the
RBCs (Red blood cells). RBCs are in charge of transporting oxygen to the body's interior organs. The virus damages the walls and linings of the alveolus and capillaries. As the walls grow, oxygen flow to red blood cells is hindered. As the wall thickens, oxygen transmission to red blood cells becomes more difficult, resulting in breathing difficulties as person's body runs out of oxygen. Furthermore, a lack of oxygen reaching the internal organs results in a deficiency in the body and impairs organ function. At this stage, body is struggling to increase the level of oxygen intake. The body's first response is for the elimination of the virus and prevent it from multiplying, but if the individual's immunity is weakened, the body is unable to do so, increasing the crisis[15]. COVID-19 can harm the heart and blood arteries, according to Dr. Laura E. Evans. This can manifest as irregular heartbeats, insufficient blood flow to the tissues, or low blood pressure requiring medication. However, it is yet unknown if the virus causes direct heart injury. In one Wuhan research, $20 \%$ of hospitalised patients showed heart disease of some kind. According to another study, $44 \%$ of patients in Intensive Care units (ICUs) had an irregular heart rhythm. COVID- 19 is connected to a higher risk of blood clotting. Although it is unclear how much clots contribute to the severity of the illness, they may raise the chance of a stroke or heart attack. The immune system attacks the foreign virus or bacterium when the body is infected. While the immune response may aid in the body's ability to fight illness, it may also harm other sections of the body. A "cytokine storm" is a severe inflammatory reaction that can occur as a result of this. To fight infection, defence cells produce cytokines, but too much of these can cause issues in the body. -During COVID-19, a lot of the damage in the body is related to what we call sepsis syndrome, which is caused by complex immunological reactions,I Evans said. -The infestation can cause a severe inflammatory reaction in the body, affecting the operation of several organ systems.l Another aspect of the immune response is that no cases of COVID-19 have been reported in children below the age of nine. Scientists are unsure whether little children are unwell or if their symptoms are so slight that they aren't noticed and no one recognises. Children also had a milder sickness than adults at the time of other diseases, Infections such as Pneumococcal disease and measles according to Cherry. These could be due to the fact that A "straightforward immune response" is seen in children, but older individuals can occasionally have a "over-reaction," according to him. During infections, it is this overactive immune reaction that causes some of the loss. Cherry stated, -There was signs of this happening during the SARS outbreak, and I assume it's happening again [with COVID-19][16].॥(See Image 1) 


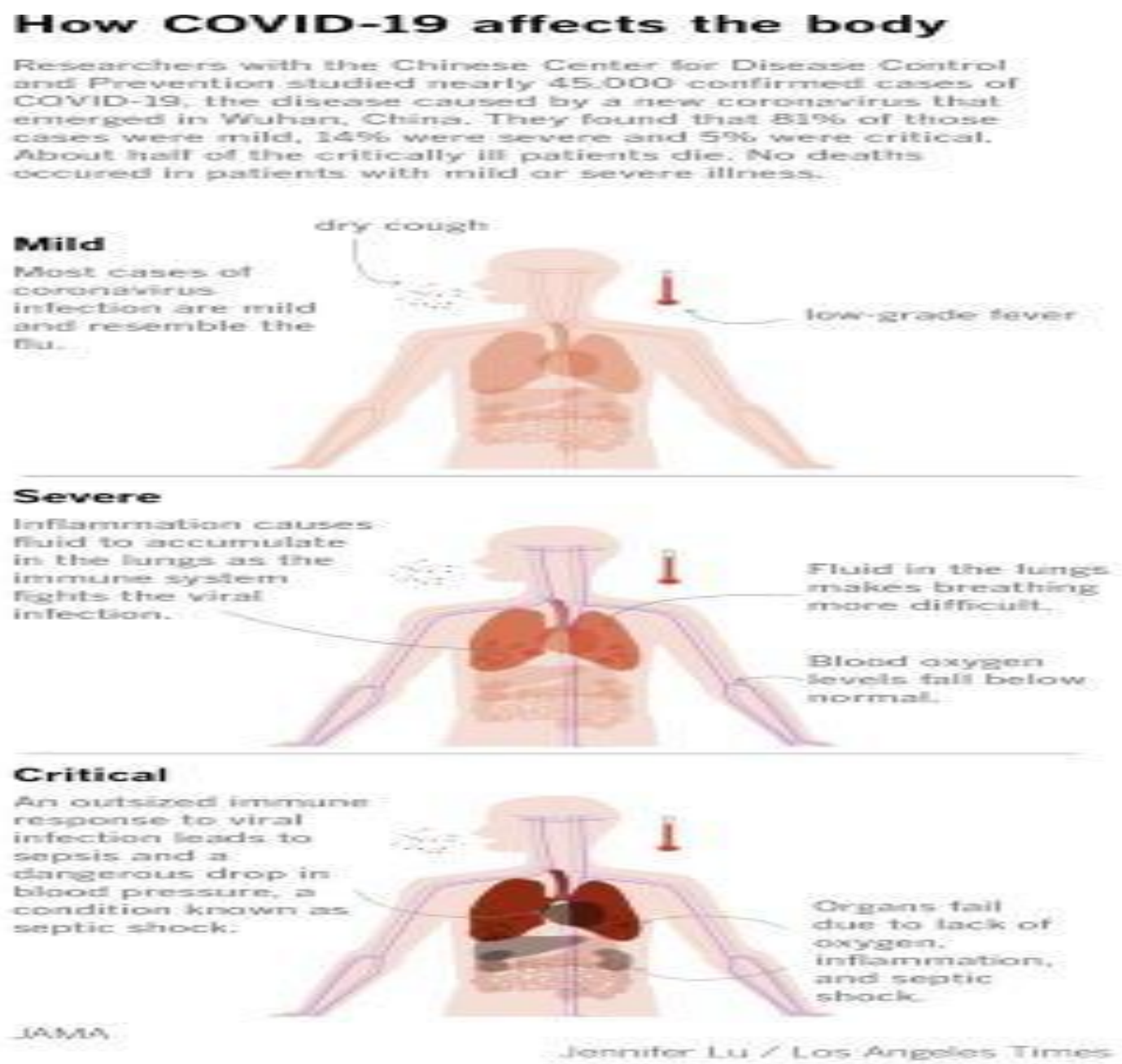

Figure 1

\section{COVID AFFECTING DIFFERENT AGE GROUPS OF PEOPLE}

The COVID-19 reveals a rise in frequency of instances and a higher risk of serious sickness as people get older, similar to the 2003 SARS pandemic. The age disparity in cases reported, which has maintained since the outbreak, could be related to children's lower infection susceptibility, lower risk of developing disease following infection, or a combination of both, when compared to adults. Recognizing the importance of age in SARS-CoV-2 transmission and illness seventy is crucial for assessing the expected global disease burden and assessing the anticipated influence of social-distancing initiatives, particularly those targeted at schools, on SARS-CoV2 transmission. For starters, the vulnerability to SARS-CoV2 infection varies by age, therefore children are less likely than adults to become infected after coming into contact with an infectious person. This would reduce the number of cases among children. Immunological cross-protection against other types of threats of corona viruses or protection from a recent infection that isn't specific with other pulmonary viruses, which children are more likely to contract than adults, could explain the reduced vulnerability. Direct indication of a drop in SARS-CoV2 susceptibility in children is mixed 5, 6, 7 but if true, it could mean less transmission in the broader population.

In addition to the aforementioned, when children are infected, they are more likely than adults to have moderate or no symptoms. Infections that cause visible symptoms lead to clinical instances, encouraging the sufferer to seek medical help. An infection that which do not cause a clinical condition may be completely asymptomatic or paucisymptomatic, meaning it causes modest symptoms that go undiagnosed or unreported despite their presence. Because asymptomatic and paucisymptomatic infections are more likely to go unnoticed than clinical infections, they are referred to as "subclinical infections," whereas the "clinical fraction" refers concerning the proportion of illnesses that occur at different ages that result in clinical symptoms. SARS and other respiratory virus infections have exhibited age-related severity changes. Among those cases reported for Covid-19, there are considerable signals of age dependence in severity and mortality $[18,19,20]$, which could extend to age-dependent severity and likelihood of clinically reportable symptoms more broadly. The number of cases recorded among children will be reduced if infected youngsters are less likely to acquire clinical symptoms. However, as has been established for influenza, children with subclinical symptoms may still be capable of transmitting the virus to others, albeit at lower rates than fully symptomatic adults[21].

Furthermore, differences in contact patterns among individuals of various ages, as well as setting-specific disparities in age distribution, alter the projected number of cases in each age group. If all other aspects are equal, children create great social ties than adults, and hence should contribute more to transmission than adults[22,23]. If the role of children has a major impact on the number of infections or cases, epidemic patterns and total impact of COVID-19 epidemics could differ dramatically between countries with different age 
distributions[17]. (See Img 2.1 and Img 2.2).

Because of the higher contact rates among children, school closures are regarded a key intervention for respiratory illness epidemics[22], albeit the impact of school closure is contingent on the engagement of children in transmission. The unique setting of SARS-CoV-2 in Wuhan, China, could have resulted in a skewed age distribution, and assortative mixing among adults could have reduced transmission to children in the very early stages of the outbreak, because early cases were concentrated in people over 40 years of age24. COVID- Working-age tourists entering China may have begun 19 outbreaks outside of China[25,26], resulting in an oversupply of adults in the early stages of local epidemics. In both situations, school closures may have lowered transmission among children even more, but to what extent is unknown[17]

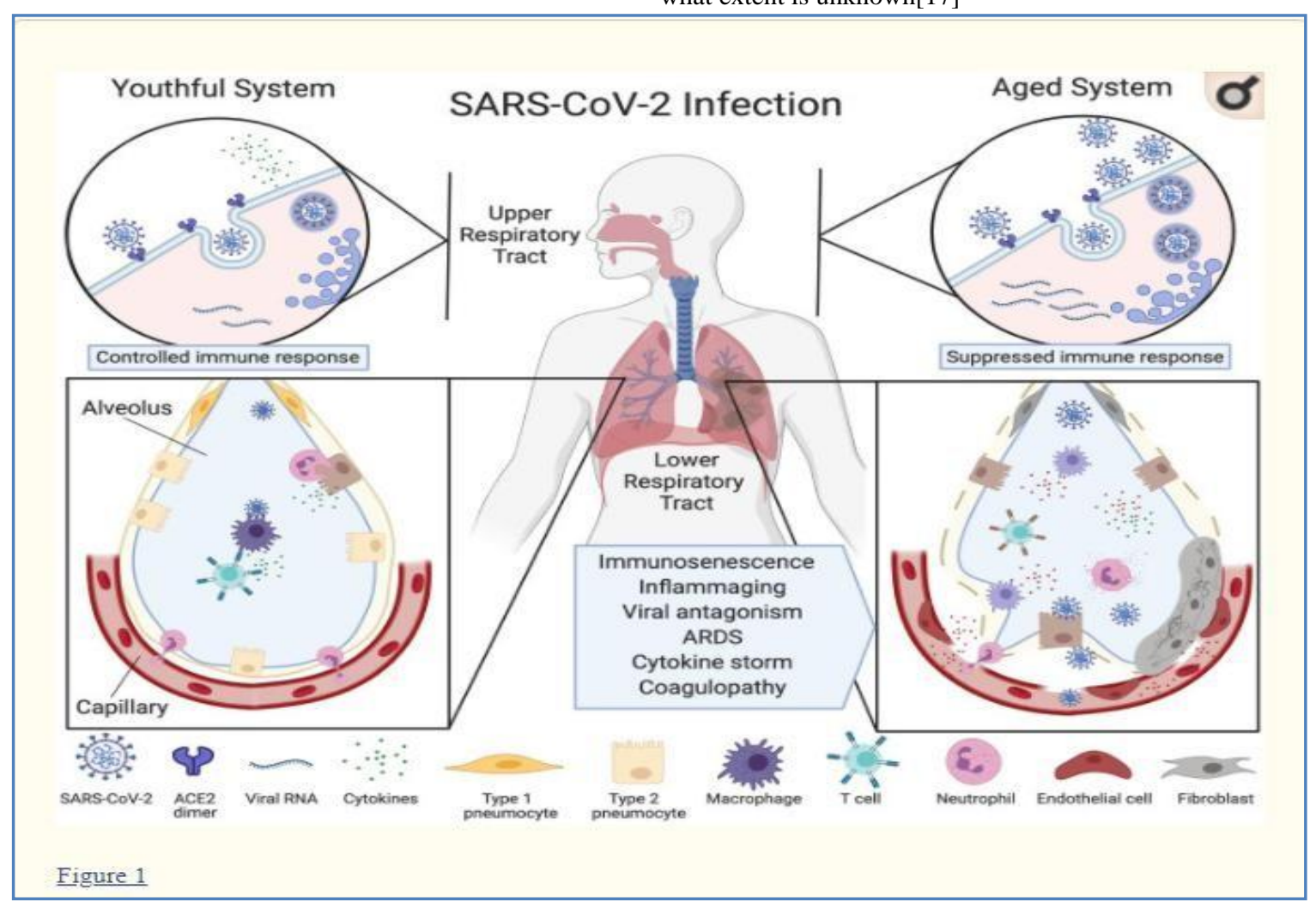

Figure 2.1: Simple Difference between Youthful System and an Old Aged System

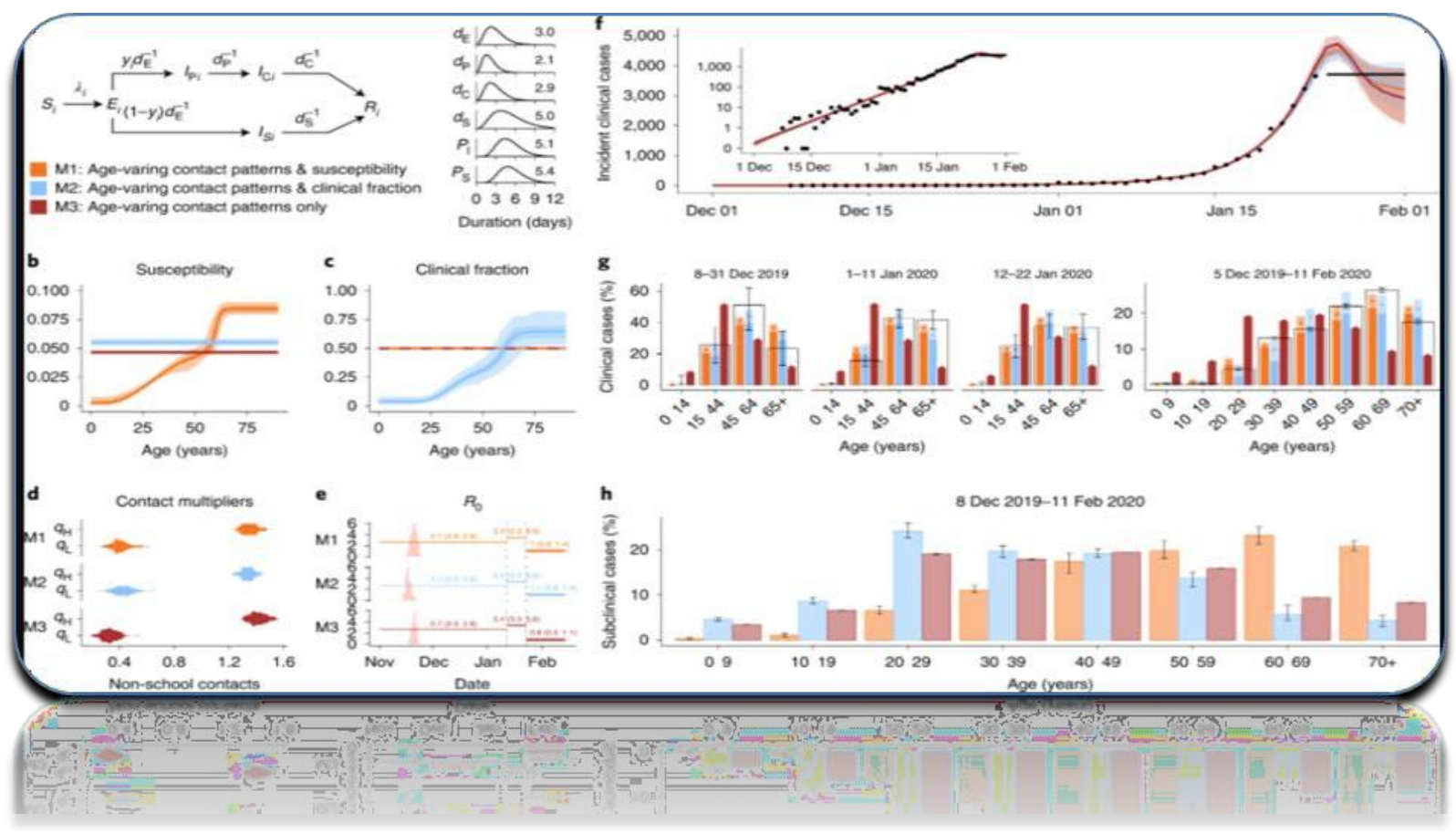

\section{PREVENTIVE MEASURE}

Figure 2.2: About the Relation BW Different Age Group and Covid 


\section{INTRODUCTION TO VACCINES WHAT ACTUALLY A VACCINE IS?}

A vaccine a chemical which is injected into the human

body to prevent any infection or to treat illness caused by a specific microbe, that is an infection leading living being, like an infection, tiny organisms or parasites. The vaccination instructs the body on how to protect itself from the microorganism by producing a safe reaction. Contrast to conventional pharmaceuticals, vaccines is biologics since they are made from living organisms. Particularly, vaccines are made of components from a pathogen they can induce a defencive impact through one to three small doses. Immunity lasts for and extended period, from one year to lifetime.

\section{For What Reason Vaccination is Required?}

There is an unprecedented requirement to produce and distribute enough secure and effective vaccine to immunise an extremely large number of people (SARS-CoV-2)[28] to save the entire global society from the long-term threat of severe acute respiratory syndrome-corona-virus 2 morbidity and mortality. Well the most appropriate answer would be as COVID-19 has some serious life threatening complications and has already affected millions of life and still going so there is no surety that it won't affect among us. And if that happen, it can pass the disease to other people around us.

Also, The masks, Social distancing, sanitizer just helps to decrease the chances for you. It doesn't eliminate it, but if with these measures if we also take vaccine it's a total full protection as the vaccine will work with your immune system and will make sure to fight the virus whenever you are exposed. For example, how India got polio free with the help of Oral Polio Vaccine (OPV).

In this case vaccine did play a huge role. There are also some advantages after you have been vaccinated fully like you can gather among other vaccinated people, you don't have to quarantine after this and also no need to test if there is no symptoms. Though there are still some measures a person should take among unvaccinated people and where there are senior citizens.

\section{Allocation of Safe and Effective COVID- 19 Vaccine}

In anticipation of the results of clinical testing, there have been encouraging agreements to produce corona-virus disease 2019 (COVID19) vaccination all over the world. Every month without a COVID-19 vaccine is costly to global public health as well as the economy, developing it practically difficult to spend too much money on vaccine trials, development, and manufacture. AstraZeneca intended to market a Covid-19 vaccine developed by Oxford University in United Kingdom, in conjunction with intention of "By September 2020, 30 million doses of the vaccine will be available in the UK, with a aspirations of 100 million doses by the close of the year. " [29].

AstraZeneca has committed to producing 2 billion doses in total, including a licence from India's Serum Institute for the delivery of 1 billion doses, with at least 400 million doses accessible towards the conclusion of the year 2020, mostly for low- and middle-income countries. While such planning was positive in the event that a viable vaccine was discovered, there are huge allocation issues ahead. We explain these issues in this paper and suggest a framework for dealing with them[30].

Because of the pandemic's global demand for vaccinations and its extensive geographic spread, more than one feasible vaccination strategy is required. Collaboration between pharmaceutical and biotech firms many of which are working on a range of vaccine strategies, will be essential (1). The entire process of developing an effective SARS-CoV-2 vaccine will need unprecedented collaboration between industry, government, and academia, with each providing their particular set of capabilities. One recent example of a public-private collaboration is the ACTIV (Accelerating COVID-19 Therapeutic Interventions and Vaccines) project. This programme, which ties all together the capabilities of all sectors at a time of global emergency, is led by the National Institutes of Health (NIH) in the United States.

A framework for collaboratively conducting harmonised, randomised controlled vaccination studies of efficacy. This method intends to collect necessary security and effectiveness facts for numerous vaccines in development at the same time, allowing different vaccine platforms and COVID-19 products to be licenced and distributed more swiftly (coronavirus disease 2019) [28]. (See Img 3) 


\section{Vaccinations by location \\ From Our World in Data · Last updated: 2 days ago}

Worldwide

\begin{tabular}{l|l|l} 
Doses given & Fully vaccinated & $\%$ of population fully vaccinated \\
$\begin{array}{l}665 \mathrm{M} \\
+11.4 \mathrm{M}\end{array}$ & $\begin{array}{l}144 \mathrm{M} \\
+2.09 \mathrm{M}\end{array}$ & $1.8 \%$ \\
\hline
\end{tabular}

\begin{tabular}{|c|c|c|c|}
\hline Location & $\begin{array}{l}\text { Doses } \\
\text { given } \downarrow\end{array}$ & $\begin{array}{l}\text { Fully } \\
\text { vaccinated }\end{array}$ & $\begin{array}{l}\% \text { of population fully } \\
\text { vaccinated }\end{array}$ \\
\hline 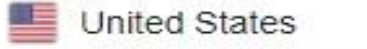 & $165 \mathrm{M}$ & $61.4 \mathrm{M}$ & $18.7 \%$ \\
\hline China (Mainland) & $140 \mathrm{M}$ & - & - \\
\hline$=$ India & 79.1M & $10.4 \mathrm{M}$ & $0.8 \%$ \\
\hline 들 United Kingdom & $36.9 \mathrm{M}$ & $5.38 \mathrm{M}$ & $8.1 \%$ \\
\hline Q. Brazil & $21.1 \mathrm{M}$ & $4.6 \mathrm{M}$ & $2.2 \%$ \\
\hline
\end{tabular}

This data shows the total number of doses given in each location. Since some vaccines require more than one dose, the number of fully vaccinated people is likely to be lower. '+' shows data reported yesterday . About this data

\section{Figure 3: Coronavirus Stats and Cases}

\section{How does the vaccine work?}

Both the Pfizer-BioNTech and Moderna vaccines use mRNA technology. mRNA is constantly used by your body's cells to generate the many proteins that you need to stay healthy. The mRNA in the two-part Coronavirus vaccinations tells your cells to make the spike protein that's unique to the new Covid. The spike protein can be detected on the virus's surface. This spike protein attaches to and enters a cell wall in your body. After you receive your Coronavirus vaccine, your immune system analyses information about the spike protein and creates an immune reaction to it. This includes the production of antibodies [31]. If you are exposed to the new Covid in any way, your immune system will utilise this knowledge to react and protect you from infection. This can assist you avoid becoming incapacitated as a result of Covid. After the second dosage of vaccine, this immunity stage takes about 2 weeks. As a result, you can acquire the new Covid you're presented with as soon as you have your vaccine.

Why do we need 2 doses?

When only one dosage of the Pfizer-BioNTech and Moderna vaccines is given, the immune response is mild, but when a second dose is added, the immune response is high. Later, it was revealed that the first dosage of vaccine only begins the process of building up virus protection, whereas the second dose completes the process and strengthen the protection and thus the ultimate shield from Covid. Apart from these two vaccine there are some other vaccine which are still under development requires two doses like Oxford-AstraZeneca, Novavax, Sputnik $\mathrm{V}$, Coronavac.

Also there is just one Covid-19 vaccine that requires only one dose and that is Johnson \& Johnson.

How much time to wait between doses?
For Pfizer-BioNtech, you will have to take second dose 3 weeks after the first dose and for Moderna you need to wait for 4 weeks after taking first shot .

Also for the under development vaccines:-

Oxoford-AstraZeneca vaccines have to 8 to 12 weeks apart, Novavax vaccine 3 weeks apart, Sputnik V 3 weeks apart, Coronavac 1 month apart.

Why is there a need for waiting?

The first dose assists with preparing your body's defence system It familiarises this with the spike protein and gives it time to mount a little immune reaction before the second injection. It will take time for this interaction to mature properly. It's also vital not to have your second dose too soon after the first, as this may compromise the vaccine's efficacy[32].

Who should not take Vaccine's shots? (see Img 4)

\section{COVAXIN}

- Have any history of allergies

- Those who are immunologic or on suppressive medications

- Those who have a fever

- Are on a blood thinner or have a bleeding issue

- Are pregnant or breastfeeding, as the vaccine's effects on pregnant women and nursing moms have not been researched.

- Received a second corona-virus vaccine.

- Any other terrible health problems in accordance with the vaccine supervising health-care officer [33]. 


\section{COVISHIELD}

- People who have had a severe allergic reaction to any of the vaccine's ingredients, which include L-Histidine hydrochloride monohydrate, Disodium edetate dihydrate (EDTA), and $\mathrm{H} 2 \mathrm{O}$ for injection.
- In case you have an allergic reaction to the first dose of Covishield, don't take it again. [33]

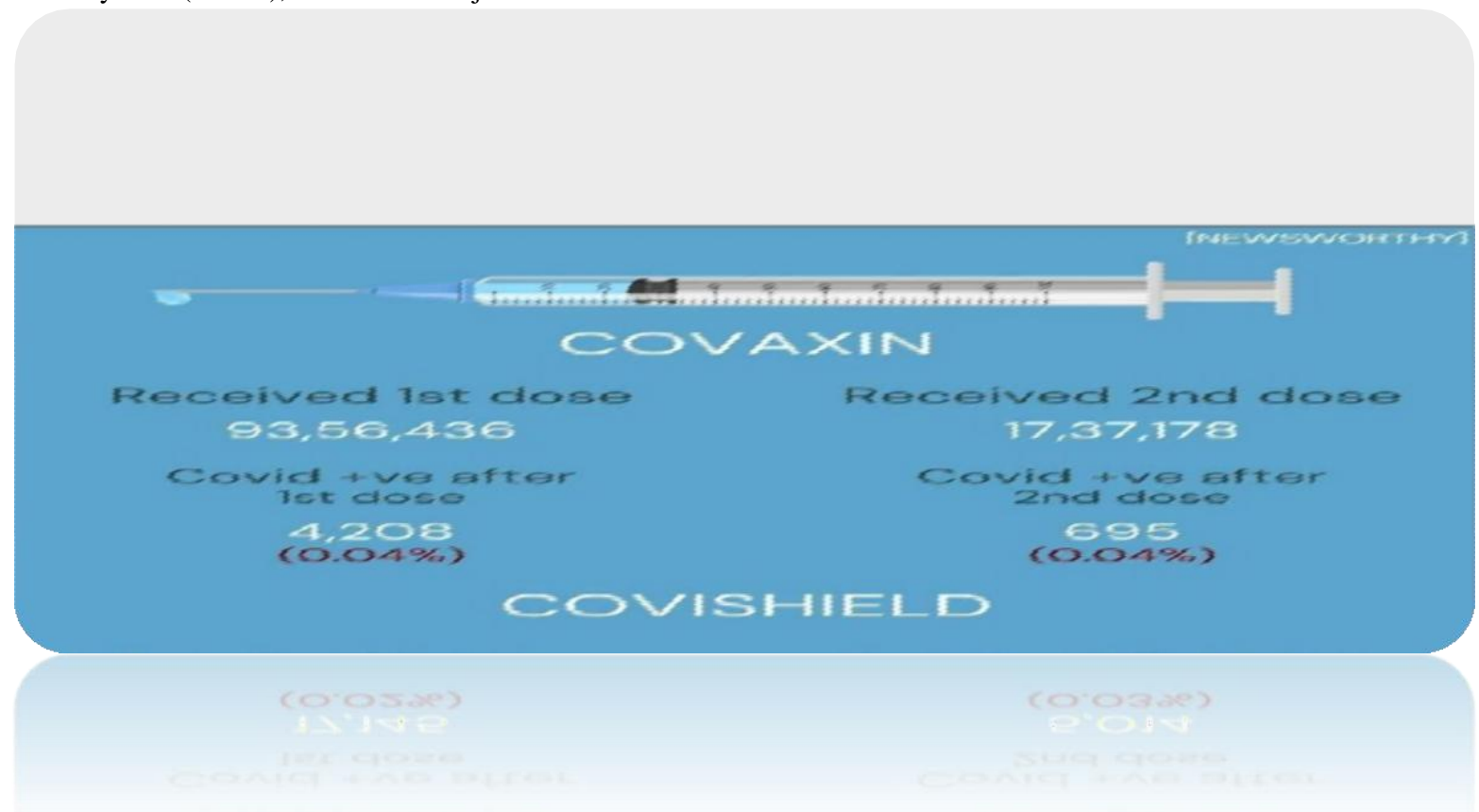

Figure 1: Rough figures between 2 vaccines

\section{Side effects of the vaccine}

Well nothing in this world is perfect, so there are some possible side effects which are just general reminder that your body is actually building the protection. It's just the body immune response to the vaccination and people should not be get discouraged by it. Tiredness, headache, muscle pain, fever, and nausea are common adverse effects. The side effects can be more intense after you take your second shot. A person is fully immunised approximately 2 weeks after receiving the second dose. Also majorly people who had covid in past and younger people experience these side effects much as compare to others[33].

\section{CONCLUSION}

After reading so many research articles,we concluded that the first wave of Covid-19 affects various age group mainly above 55-60,which further affects the human body and thus affecting the humanity.

This research looked into the effects of COVID-19, which was released in a relatively short period of time. The goal is to bring academic and policy debates together and to aid future research. Before diving into COVID-19's ramifications, we documented the data sources about its affect on humanity, human body and people of different age groups. COVID-19 is a global societal hazard, as we previously stated. Societal hazards, according to the WHO, are natural or mankind-caused threats which can impact a significant part of the population of human, infecting above 46 million people in 235 countries and areas throughout the world (2020). However, In humans, Covid 19 seems to have an effect on the respiratory tract and thus destroying the lungs of the people in suffering, mainly above the age of 55-60. The report also looked at several studies on preventive interventions, particularly in terms of their effectiveness in COVID-19 vaccine allocation. Going forward, vaccine doses and sides effects play a key role in vaccination.

Technology can help in this scenario by making various models which can predict the next wave or how much time we need to deal with these waves. Technology like i)AI; ii)Analysis; iii)Python; iv)R-studio; v)Anaconda and many more can be very beneficial. Future research will explore about the empirical technical information regarding the same.

\section{REFERENCES}

[1] Goyal, L., \& Arora, N. Deep Transfer Learning Approach for Detection of Covid-19 from Chest X- Ray Images. International Journal of Computer Applications, 975, 8887.

[2] Coronavirus 2019-nCoV, CSSE . Coronavirus 2019-nCoV Global Cases by Johns Hopkins CSSE. (Available from: https://gisanddata.maps.arcgis.com/apps/opsdas hboard/index.html\#/bda7594740fd40299423467b48e 9ecf6)

[3] Coronaviruses closely related to the pandemic virus discovered in Japan and Cambodia https://www.nature.com/articles/d41586-020-03217-0

[4] Kuldeep Dhama, Sharun Khan, Ruchi Tiwari, Shubhankar Sircar, Sudipta Bhat, Yashpal Singh Malik, Karam Pal Singh, Wanpen Chaicumpa, D. Katterine Bonilla-Aldana, Alfonso J. Rodriguez- Morales.(2021). Coronavirus Disease 2019-COVID-19. https://cmr.asm.org/content/33/4/e00028- 20.full\#sec-14/

[5] Huang C., Wang Y., Li X., Ren L., Zhao J., Hu Y., Zhang L., Fan G., Xu J., Gu X., et al. Clinical features of patients infected with 2019 novel coronavirus in Wuhan, China. Lancet. 2020;395:497-506. doi: 10.1016/S01406736(20)30183-5. [PMC free article] [PubMed] [CrossRef] 
[Google Scholar]

[6] Zhou P., Yang X.-L., Wang X.G., Hu B., Zhang L., Zhang W., Si R.H., Zhu Y., Li B., Huang C.-L., et al. A pneumonia outbreak associated with a new coronavirus of probable bat origin. Nature. 2020;579:270-273. [PMC free article] [PubMed] [Google Scholar]

[7] Guan W.-J., Ni Z.-Y., Hu Y., Liang W.-H., Ou C.-Q., He J.X., Liu L., Shan H., Lei C.-L., Hui D.S., et al. Clinical characteristics of coronavirus disease 2019 in China. New Engl. J. Med. 2020 doi: 10.1056/NEJMoa2002032. [PMC free article] [PubMed] [CrossRef] [Google Scholar]

[8] Guo Y.-R., Cao Q.-D., Hong Z.-S., Tan Y.-Y., Chen S.-D., Jin H., Tan K.-S., Wang D.-Y., Yan Y. The origin, transmission and clinical therapies on coronavirus disease 2019 (COVID-19) outbreak-An update on the status. Mil. Med Res. 2020;7:1-10. doi: 10.1186/s40779-020-00240-0. [PMC free article] [PubMed] [CrossRef] [Google Scholar]

[9] Lu R., Zhao X., Li J., Niu P., Yang B., Wu H., Wang W., Song H., Huang B., Zhu N., et al. Genomic characterisation and epidemiology of 2019 novel coronavirus: Implications for virus origins and receptor binding. Lancet. 2020;395:565-574. doi: 10.1016/S0140-6736(20)30251-8. [PMC free article] [PubMed] [CrossRef] [Google Scholar] Tang X., Wu C., Li X., Song Y., Yao X., Wu X., Duan Y., Zhang H., Wang Y., Qian Z., et al. On the origin and continuing evolution of SARS-CoV-2. Natl. Sci. Rev. 2020 doi: 10.1093/nsr/nwaa036. [CrossRef] [Google Scholar]

[10] Zhang L., Liu Y. Potential interventions for novel coronavirus in China: A systematic review. J. Med. Virol. 2020;92:479-490. doi: 10.1002/jmv.25707. [PMC free article] [PubMed] [CrossRef] [Google Scholar]

[11] Lee N., Hui D.S., Wu A., Chan P.K.S., Cameron P., Joynt G., Ahuja A.T., Yung M.Y., Leung C., To K., et al. A major outbreak of severe acute respiratory syndrome in Hong Kong. New Engl. J. Med. 2003;348:1986-1994. doi: 10.1056/NEJMoa030685. [PubMed][CrossRef] [Google Scholar]

[12] Gautret P., Lagier J.-C., Parola P., Hoang V.T., Meddeb L., Mailhe M., Doudier B., Courjon J., Giordanengo V., Vieira V.E., et al. Hydroxychloroquine and azithromycin as a treatment of COVID-19: Results of an open-label nonrandomized clinical trial. Int. J. Antimicrob. Agents. 2020:105949. doi:10.1016/j.ijantimicag.2020.105949. [PMC free article] [PubMed] [CrossRef] [Google Scholar]

[13] Nguyen T.M., Zhang Y., Pandolfi P.P. Virus against virus: A potential treatment for 2019-nCov (SARS- CoV-2) and other RNA viruses. Cell Res. 2020;30:189-190. doi: 10.1038/s41422-020-0290-0. [PMC free article] [PubMed] [CrossRef] [Google Scholar]

[14] Yoshihisa Kashima, Simon Dennis, Andrew Perfors, Simon M. Laham (2021). Culture and global societal threats: COVID-19 as a pathogen threat to humanity. https://journals.sagepub.com/doi/full/10.1177/136843 0220982075/

[15] [15] CORONAVIRUS COMPLICATIONS: HOW DOES COVID 19 AFFECT YOUR BODY? written by Narayana Health. https://www.narayanahealth.org/blog/how-covid-19affect-your-lungs/

[16] Here's What Happens to the Body After Contracting the
New Coronavirus Written by Shawn Radcliffe on April 30, 2020 - Fact checked by Dana K. Cassell https://www.healthline.com/health-news/heres-whathappens-to-the-body-after-contracting-thecoronavirus\#Heart-and-blood-vessels

[17] Here's What Happens to the Body After Contracting the New Coronavirus Written by Shawn Radcliffe on April 30, 2020 - Fact checked by Dana K. Cassell https://www.healthline.com/health-news/heres-whathappens-to-the-body-after-contracting-thecoronavirus\#Heart-and-blood-vessels

[18] Here's What Happens to the Body After Contracting the New Coronavirus Written by Shawn Radcliffe on April 30, 2020 - Fact checked by Dana K. Cassell https://www.healthline.com/health-news/heres-whathappens-to-the-body-after-contracting-thecoronavirus\#Heart-and-blood-vessels

[19] Nicholas G. Davies, Petra Klepac, Yang Liu, Kiesha Prem, Mark Jit, CMMID COVID-19 working group \& Rosalind M. Eggo (2020). Age-Dependents effects in the transmission and control of COVID-19 epidemics. https://www.nature.com/articles/s41591- 020-0962-9

[20] Galanti, M. et al. Rates of asymptomatic respiratory virus infection across age groups. Epidemiol. Infect. 147, e176 (2019).

[21] Zhou, F. et al. Clinical course and risk factors for mortality of adult inpatients with COVID-19 in Wuhan, China: a retrospective cohort study. Lancet 395, 1054-1062 (2020).

[22] Riccardo, F. et al. Epidemiological characteristics of COVID-19 cases in Italy and estimates of the reproductive numbers one month into the epidemic. Preprint at http://medrxiv.org/lookup/doi/10.1101/2020.04.08. 20056861 (2020).

[23] Van Kerckhove, K., Hens, N., Edmunds, W. J. \& Eames, K. T. D. The impact of illness on social networks: implications for transmission and control of influenza. Am. J. Epidemiol. $178,1655-1662$ (2013).

[24] Cauchemez, S., Valleron, A.-J., Boëlle, P.-Y., Flahault, A. \& Ferguson, N. M. Estimating the impact of school closure on influenza transmission from Sentinel data. Nature 452, 750-754 (2008).

[25] Eames, K. T. D., Tilston, N. L., Brooks-Pollock, E. \& Edmunds, W. J. Measured dynamic social contact patterns explain the spread of H1N1v influenza. PLoS Comput. Biol. 8 , e1002425 (2012).

[26] Huang, C. et al. Clinical features of patients infected with 2019 novel coronavirus in Wuhan, China. Lancet 395 , 497-506 (2020). CAS Article Google Scholar

[27] Koh, A. Singapore COVID-19 Cases (2020); http://alexkoh.net/covid19/ (accessed 4 March 2020).

[28] Data Science for COVID-19 (DS4C) (2020); https://kaggle.com/kimjihoo/coronavirusdatas et (accessed 13 March 2020).

[29] http://www.biotech.ca/wpcontent/uploads/2016/04/vaccines_1_2010-1.pdf

[30] Lawrence Corey 1,2, John R. Mascola 3 , Anthony S. Fauci 4 , Francis S. Collins https://science.sciencemag.org/content/sci/368/6494/9 48.full.pdf 
[31] Laurence S. J. Roope, John Buckell, Frauke Becker,Paolo Candio, Mara Violato,John Buckell,Frauke Becker,Paolo Candio, Mara Violato, Jody L. Sindelar,Adrian Barnett, Raymond Duch \& Philip M. Clarke https://link.springer.com/article/10.1007/s41669-02000228-5

[32] Herper M. AstraZeneca lays out plan for producing 2 billion doses of Covid-19 vaccine, if it works. STAT. 2020. https://www.statnews.com/2020/06/04/astrazen eca-laysout-plan-for-producing-2-billion-doses-ofcovid-19vaccine-if-it-works/. Accessed 12 Aug 2020.

[33] Why Do You Need Two Doses for Some COVID-19 Vaccines? https://www.healthline.com/health/why- twodoses-of-covid-vaccine
[34] Why Do You Need Two Doses for Some COVID-19 Vaccines? Medically reviewed by Meredith Goodwin, MD, FAAFP - Written by Jill Seladi-Schulman, Ph.D.

[35] RESTRICTED USE IN EMERGENCY SITUATION OF COVID-19 SARS-CoV-2 VACCINE BY BHARAT BIOTECH

https://www.bharatbiotech.com/images/covaxin/cova xinfact-sheet.pdf

[36] Facts and Figures from:https://apps.who.int/iris/bitstream/handle/10665/3329 71/nCoVsitrep01Jul2020-eng.pdf

[37] Kakde, A., Sharma, D., \& Arora, N. (2020). Optimal Classification of COVID-19: A Transfer Learning Approach. Int. J. Comput. Appl., 176(20), 25-31. 